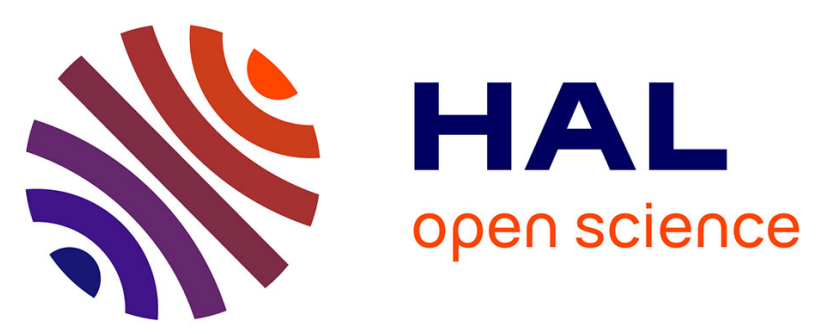

\title{
Asymptotic Analysis and Design of Iterative Receivers for Non Linear ISI Channels
}

Bouchra Benammar, Nathalie Thomas, Charly Poulliat, Marie-Laure

Boucheret, Mathieu Dervin

\section{- To cite this version:}

Bouchra Benammar, Nathalie Thomas, Charly Poulliat, Marie-Laure Boucheret, Mathieu Dervin. Asymptotic Analysis and Design of Iterative Receivers for Non Linear ISI Channels. 8th International Symposium on Turbo Codes \& Iterative Information Processing - ISTC (2014), Aug 2014, Bremen, Germany. pp. 173-177. hal-01147241

\section{HAL Id: hal-01147241 https://hal.science/hal-01147241}

Submitted on 30 Apr 2015

HAL is a multi-disciplinary open access archive for the deposit and dissemination of scientific research documents, whether they are published or not. The documents may come from teaching and research institutions in France or abroad, or from public or private research centers.
L'archive ouverte pluridisciplinaire HAL, est destinée au dépôt et à la diffusion de documents scientifiques de niveau recherche, publiés ou non, émanant des établissements d'enseignement et de recherche français ou étrangers, des laboratoires publics ou privés. 


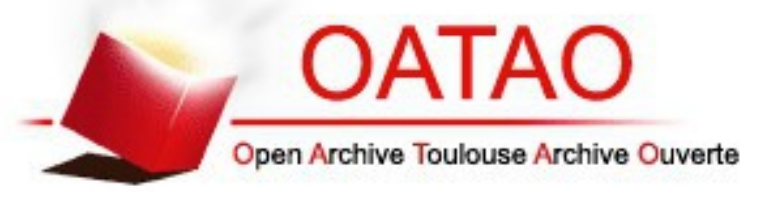

\section{Open Archive TOULOUSE Archive Ouverte (OATAO)}

OATAO is an open access repository that collects the work of Toulouse researchers and makes it freely available over the web where possible.

This is an author-deposited version published in : http://oatao.univ-toulouse.fr/ Eprints ID : 13122

To link to this article : DOI :10.1109/ISTC.2014.6955108

URL : http://dx.doi.org/10.1109/ISTC.2014.6955108

To cite this version : Benammar, Bouchra and Thomas, Nathalie and Poulliat, Charly and Boucheret, Marie-Laure and Dervin, Mathieu Asymptotic Analysis and Design of Iterative Receivers for Non Linear ISI Channels. (2014) In: 8th International Symposium on Turbo Codes \& Iterative Information Processing - ISTC (2014), 18 August 2014 - 22 August 2014 (Bremen, Germany).

Any correspondance concerning this service should be sent to the repository administrator: staff-oatao@listes-diff.inp-toulouse.fr 


\title{
Asymptotic Analysis And Design Of Iterative Receivers For Non Linear ISI Channels
}

\author{
Bouchra Benammar*, Nathalie Thomas*, Charly Poulliat*, Marie-Laure Boucheret*, Mathieu Dervin ${ }^{\dagger}$ \\ *University of Toulouse, INPT-ENSEEIHT/IRIT, Toulouse France \\ \{bouchra.benammar, nathalie.thomas, charly.poulliat, marie-laure.boucheret\}@enseeiht.fr \\ $\dagger$ Thales Alenia Space, Toulouse, France \\ \{mathieu.dervin@thalesaleniaspace.com\}
}

\begin{abstract}
In this paper, iterative receiver analysis and design for non linear satellite channels is investigated. To do so, an EXtrinsic Information Transfer (EXIT) chart-based optimization is applied using two major assumptions: the equalizer outputs follow a Gaussian Mixture distribution since we use non-binary modulations and partial interleavers are used between the Low Density Parity Check (LDPC) code and the mapper. Achievable rates, performance and thresholds of the optimized receiver are analysed. The objective in fine is to answer the question: Is it worth optimizing an iterative receiver for non linear satellite channels?
\end{abstract}

Keywords-iterative equalization, Volterra series, LDPC code design, optimization

\section{INTRODUCTION}

The advent of turbo-codes [1], and the reinvention of Gallager codes (LDPC) enabled reaching near Shannon limit performance. Hence, designing capacity approaching codes has gained a wide attention for both memoryless and memory channels in the last decade. This paper addresses the issue of designing LDPC codes for non linear memory channels, and more specifically non linear Volterra channels. In fact, satellite communications are subject to non linear Inter Symbol Interference (ISI). This interference results from the use of power amplifiers near to their saturation region for a better energy efficiency. Thus, input satellite signals with high dynamics -either because of the transmit filters or because of non constant amplitude constellations- are non linearly amplified and hence distorted. This phenomenon is a limiting issue for satellite standards such as DVB-S2 [2] which proposed non-constant amplitude modulations such as 16/32 Amplitude and Phase Shift Keying (APSK). The non linear Volterra channel can be seen as a memory channel represented by a state machine, or a trellis, with non linear branch metrics. This representation allows applying optimal symbol and sequence detection techniques. Yet, joint equalization and decoding following the principle of turbo-equalization proposed in [3] can dramatically enhance system performance. A further enhancement can be achieved when the channel code is designed to best fit the equalization behaviour. Code design and optimization is however only interesting when the channel characteristics do not vary rapidly which is the case of the satellite non linear channel. A great deal of literature addressed the optimization of LDPC codes for static linear ISI channels [4] and partial response ISI channels [5]. Authors in [6] used curve fitting of the EXIT charts to design the code, by considering a serial concatenation of a block containing the detector and the variable nodes with a block of check nodes. Application to the Multiple Input Multiple Output (MIMO) over fading channels with Channel State Information (CSI) at the receiver was investigated. Another optimization approach is presented in [7] and uses a factor graph description for both the detector and the decoder. A simple derivation of the Sum Product (SP) of the overall receiver factor graph is then presented. As far as non linear satellite channels are concerned, authors in [8] optimized the non-iterative 16-APSK constellation ring ratios in order to allow for the best performance for different LDPC coding rates and spectral efficiencies. Authors in [9] investigated the performance of MAP turbo-equalization for non linear satellite channels. Yet, no thorough iterative receiver optimization for non linear channels has yet been investigated. Hence, we are interested in the analysis and the code design for turbo MAP equalization over non linear ISI satellite channels. A serial concatenation approach similar to [6] is used, but the equalizer outputs are assumed to follow a Gaussian Mixture (GM) distribution since the Gaussian approximation is not accurate for non-binary modulations. In addition, partial interleavers will be used for variable nodes of the same degree in order to allow for an independence assumption among nodes of the same degree. The rest of this paper is organised as follows: Section II presents the Volterra non linear channel with trellis representation. Section III outlines the MAP turbo equalizer where Section IV presents the concepts of EXIT charts applied to 16-APSK and LDPC variable and check nodes. Section V presents the optimization problem as well as obtained results. Section VI draws conclusions and perspectives.

\section{NONLINEAR VOLTERRA CHANNEL}

Let us consider the scheme depicted in Fig. 1. A source produces blocks of $K$ independent and identically distributed bits $\mathbf{b}=\left(b_{0}, \ldots, b_{K-1}\right) \in G F(2)^{K}$ which are encoded with a binary LDPC code with code rate $R=K / N$ producing blocks of codewords $\mathbf{c}=\left(c_{0}, \ldots c_{N-1}\right) \in G F(2)^{N}$ of length $N$. A binary LDPC code is defined by its $(N-K, N)$ parity check matrix which allows for a representation of the LDPC code using a Tanner graph involving two types of vertices, 


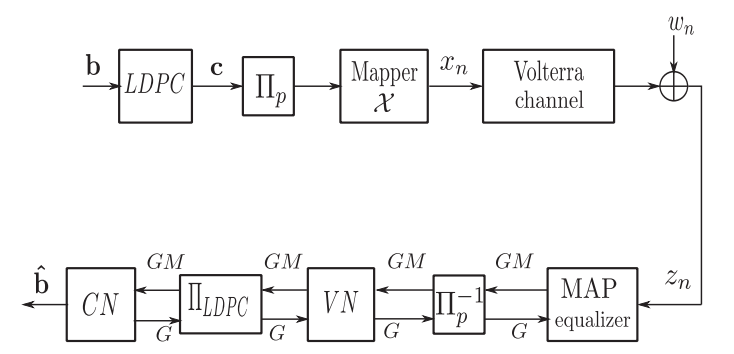

Fig. 1. Global scheme of a satellite communication channel. GM stands for quantities with a Gaussian Mixture approximation, G for Gaussian approximation

namely Variable Nodes (VN) and Check Nodes (CN). A codeword c satisfies $H \mathbf{c}^{T}=0$. A variable node $m$ (on the $m^{\text {th }}$ column of $H$ ) is related to a check node $n$ ( $n^{\text {th }}$ row of $H$ ) by edge $E_{n, m}$ when $H_{n, m}=1$. By defining $\lambda_{i}$ (resp. $\rho_{j}$ ) as the proportion of edges related to degree- $i$ VNs (resp. to degree- $j$ CNs), an LDPC code ensemble can also be characterised by the distribution polynomials of edges degree writing as follows:

$$
\lambda(X)=\sum_{i=2}^{d_{v}} \lambda_{i} X^{i-1} \quad \rho(X)=\sum_{j=2}^{d_{c}} \rho_{j} X^{j-1}
$$

where $d_{v}$ (resp. $d_{c}$ ) represents the maximum degree of variable (resp. check) nodes. Codewords are then fed to a mapping function with cardinality $M$ which converts blocks of $\log _{2}(M)$ coded bits into symbols $x_{n} \in \mathcal{X}$ where $\mathcal{X}$ is the mapping alphabet. A partial interleaver is used to interleave the incoming codewords belonging to variable nodes of the same degree (justification and details can be found in Section III). The symbols $x_{n}$ are then passed through the non linear satellite channel. The satellite power amplifier is a memoryless device that has an amplification characteristic which is only function of the amplitude of the signal to be amplified. However, due to satellite input and output filters, the satellite transponder is equivalent to a memory channel. At the receiver a Gaussian circular noise $w_{n}$ corrupts the input signal. It has been shown in [10] that the received symbols can be written as a mixture of linear and non linear ISI terms, namely:

$$
\begin{aligned}
z_{n}= & \sum_{m=0}^{v_{m}} \sum_{n_{1}=-\infty}^{\infty} \ldots \sum_{n_{2 m+1}=-\infty}^{\infty} x_{n-n_{1}} \ldots x_{n-n_{m+1}} \\
& x_{n-n_{m+2}}^{*} \ldots x_{n-n_{2 m+1}}^{*} h_{n_{1}, \ldots, n_{2 m+1}}+w_{n}
\end{aligned}
$$

where $v_{m}$ defines the decomposition order of the Volterra series, $h_{n_{1}, \ldots, n_{2 m+1}}$ are Volterra kernels and $w_{n} \sim \mathcal{N}\left(0, \sigma_{w}^{2}\right)$ is the filtered sampled additive noise. In fact, the Volterra kernels are not only function of the chain filters but also on the mapping alphabet $\mathcal{X}$. As argued in [10], non linear kernels with order greater than five have negligible contribution to the ISI terms, which yields a third order decomposition as follows:

$$
z_{n}=\sum_{i \in \mathcal{I}_{1}^{1}} h_{i} x_{n-i}+\sum_{i \in \mathcal{I}_{1}^{3}} \sum_{j \in \mathcal{I}_{2}^{3}} \sum_{k \in \mathcal{I}_{3}^{3}} h_{i j k} x_{n-i} x_{n-j} x_{n-k}^{*}+w_{n}
$$

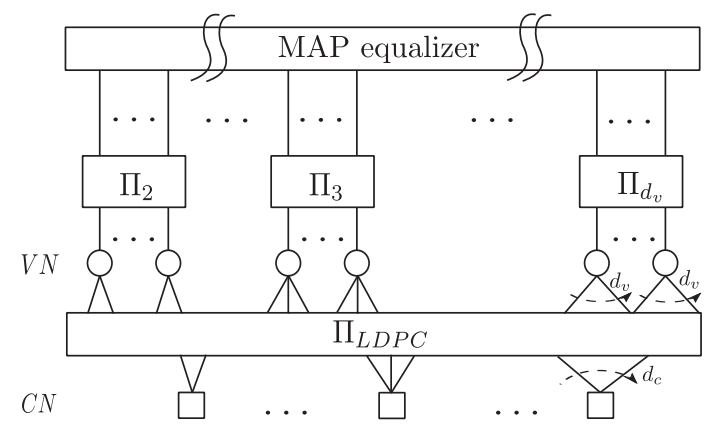

Fig. 2. Equalizer and LDPC code model

$$
=\mathcal{F}\left(\left(x_{n-k}\right)_{k \in \mathcal{I}}, x_{n}\right)+w_{n}
$$

where $\mathcal{I}_{p}^{l}$ denotes the index set of symbols at position $p$ in the $l^{\text {th }}$ order of the Volterra decomposition in (2) and $\mathcal{I}=$ $\underset{(p, l)}{\cup}\left\{\mathcal{I}_{p}^{l}\right\} \backslash\{0\}$. We assume that the Volterra decomposition is causal i.e. $\forall i \in \mathcal{I}_{p}^{l} i \geq 0$

\section{NON LINEAR CHANNEL ITERATIVE RECEIVER}

\section{A. Iterative MAP equalizer}

The non linear Volterra channel as described by (3) can be represented by trellis with a state set $\mathcal{S}$ with cardinality $|\mathcal{S}|=M^{i_{\max }+1}$ and $M$ transitions per state, where $i_{\max }=$ $\max \{i \in \mathcal{I}\}$. The branch transitions outputs are both linear and non linear functions of the memory symbols following (3). This representation allows for an optimal symbol detection based on MAP equalization. More specifically, we use the Bahl-Cocke-Jelinek-Raviv (BCJR) efficient MAP implementation. The LDPC code concatenated with a Markov process channel allows for using joint iterative equalization and decoding which yields better error rates, yet, with a significant complexity increase. More specifically, let $L_{a}\left(c_{n, i}^{\prime}\right), L\left(c_{n, i}^{\prime}\right)$ and $L_{e}\left(c_{n, i}^{\prime}\right)$ be the a priori, a posteriori and extrinsic Log Likelihood Ratios (LLRs) of the $i^{\text {th }}$ coded bit belonging to the $n^{t h}$ symbol $\left(c_{n, i}\right)$. The a posteriori LLR of coded bit $\left(c_{n, i}^{\prime}\right)$ knowing the whole received symbols sequence $\mathbf{z}=\left(z_{n}\right)_{n \in \mathbb{N}}$ writes as follows:

$$
\begin{aligned}
L\left(c_{n, i}^{\prime}\right) & =\log \frac{P\left(c_{n, i}^{\prime}=0 \mid \mathbf{z}\right)}{P\left(c_{n, i}^{\prime}=1 \mid \mathbf{z}\right)} \\
& =\log \frac{\sum_{s_{j}: s_{j, i}=0} P\left(x_{n}=s_{j}, \mathbf{z}\right)}{\sum_{s_{j}: s_{i j}=1} P\left(x_{n}=s_{j} ; \mathbf{z}\right)}
\end{aligned}
$$

where for $j \in 1, \ldots, M, P\left(x_{n}=s_{j}, \mathbf{z}\right)$ is computed following the forward/backward recursion.

\section{$B$. Iterative $L D P C$ decoder and scheduling}

We will assume that Belief Propagation (BP)-based LDPC decoding is used [11]. Besides, MAP equalization is assumed to be run independently on each group delimited by a partial interleaver i.e. transition effects are neglected. In this paper, the following scheduling will be used: a global iteration $t$ is composed of one BCJR forward-backward recursion for the 
equalizer followed by one BP iteration (one data-pass plus check-pass update) for the LDPC code. We further assume partial interleaving $\Pi_{p}$ between the channel equalizer and the LDPC code operated degree-wise, i.e. each partial interleaver is associated with the VNs set of the same degree. The assumption is similar to [6] and ensures an efficient optimization problem statement which becomes linear. Furthermore, and for independence considerations, a global interleaver $\Pi_{L D P C}$ is assumed between check and variable nodes as depicted in Fig. 2 representing the classical code ensemble interleaving. ${ }^{1}$

\section{ASYMPTOTIC CODE DESIGN USING EXIT CHARTS}

EXtrinsic Information Transfer chart is a common tool to analyse the convergence behaviour of a Soft Input Soft Output (SISO) component. For a binary input AWGN channel $Y=a X+N$, the output extrinsic LLRs follow a consistent Gaussian distribution $\mathcal{N}(\mu, 2 \mu)$ where $\mu=\frac{2 a^{2}}{\sigma^{2}}$ and $\sigma_{w}^{2}$ is the noise variance. The mutual information of LLRs following Gaussian consistent distribution writes as:

$$
J(\sigma)=1-E_{x}\left(\log _{2}\left(1+e^{-x}\right)\right)
$$

where expectation is taken with respect to $x \sim \mathcal{N}\left(\frac{\sigma^{2}}{2}, \sigma^{2}\right)$. For Gray mapped QPSK, it can be shown that the softdemapper extrinsic output LLR is a mixture of two consistent Gaussian distributions with mean and variance independent of the input a priori. As far as higher order gray-mapped modulations are concerned, the output extrinsic LLRs are Gaussian mixtures with parameters depending on the input apriori information. However, the slight impact of the apriori on the parameters of the GM distribution for medium to large Signal to Noise Ratios (SNRs), allows for a flat EXIT chart approximation at a given SNR. This approximation also holds for the 16-APSK which has a quasi gray mapping. The mutual information of LLRs following a GM distribution $\sum_{m=1}^{M_{G}} \pi_{m} \mathcal{N}\left(\frac{\beta_{m}}{2} \sigma^{2}, \beta_{m} \sigma^{2}\right)$, where $M_{G}$ is the number of mixtures, writes as follows:

$$
\Psi(\sigma)=\sum_{m=1}^{M_{G}} \pi_{m} J\left(\sqrt{\beta_{m}} \sigma\right)
$$

In order to optimize the LDPC code for a given receiver structure, mutual informations at the input and output of the equalizer, variable and check nodes are evaluated. The objective is to optimize the edge-degree distribution polynomials $\lambda(X)$ and $\rho(X)$ in order to achieve the highest rate under some set of constraints.

\section{A. MAP equalizer EXIT chart}

Let $I_{M A P, V}$ denote the extrinsic output mutual information of the MAP equalizer in response to an input information $I_{V, M A P}$ coming from a variable node. The EXIT curve plots for a given $\mathrm{SNR}, I_{M A P, V}=T\left(I_{V, M A P}\right)$, where $T$ is a non-decreasing function. Computations of the MAP EXIT curves are analytically complex, but numerical simulations can

\footnotetext{
${ }^{1}$ Note that the proposed approach is not a Multi-Edge Type (MET) due to the uniform interleaving of edges of the LDPC code.
}

TABLE I

GAUSSIAN MiXTURE PARAMETERS

\begin{tabular}{|ll|}
\hline Gaussian Mixture parameters & \\
\hline$\beta_{5}=\beta_{4}=0.33$ & $\beta_{6}=\beta_{3}=0.66$ \\
$\beta_{7}=\beta_{2}=1.29221$ & $\beta_{8}=\beta_{1}=1.9$ \\
\hline$\pi_{5}=\pi_{4}=0.25$ & $\pi_{6}=\pi_{3}=0.125$ \\
$\pi_{7}=\pi_{2}=0.0625$ & $\pi_{8}=\pi_{1}=0.0625$ \\
\hline
\end{tabular}

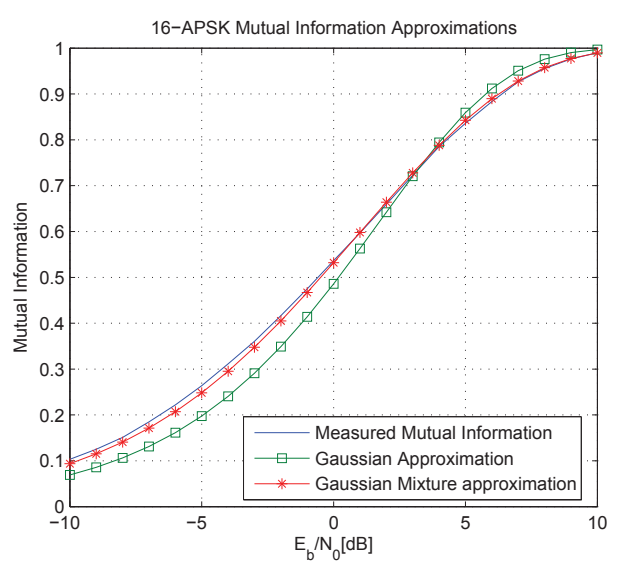

Fig. 3. Mutual Information Vs SNR for 16APSK AWGN soft demapper output

provide good approximations of the real curves. These EXIT charts are also exploited in finding an upper bound to the achievable rate of the iterative receiver. The upper bound, is given by the so called area theorem [12] which states that for serially concatenated codes and Binary Erasure Channel (BEC), the achievable rate $R$ of an outer code, is upperbounded by the integral of the inner code transfer curve $T$ i.e. $R \leq \int_{0}^{1} T(I) d I$ when the latter is a rate 1 code. It is widely observed that this upper bound is a good approximation for other channel models. At perfect a priori information, the performance of the MAP joins that of the soft demapper for a 16-APSK Bit Interleaved Coded Modulation (BICM). Thus, the GM distribution in (6) is a good approximation of the mutual information of the MAP equalizer output LLRs. The mutual information of 16-APSK at the output of the AWGN soft demapper is computed for each SNR at input information $I_{A}=1$. The parameters of the Gaussian mixture $\left(\pi_{m}, \beta_{m}\right)$ were estimated using a Maximum-Likelihood with Expectation Maximisation (EM) algorithm and are presented in table I. The inverse of function $\Psi$ is computed using trustregion-reflective non linear least square approximation, after a coarse estimation of the turning point. $\Psi^{-1}$ writes as follows:

$$
\Psi^{-1}(I)= \begin{cases}a_{1} I^{4}+a_{2} I^{3}+a_{3} I^{2}+a_{4} I & \text { if } I<0.5654 \\ -b_{1} \log \left(-b_{2}(I-1)\right)+b_{3} I & \text { if } I \geq 0.5654\end{cases}
$$

where $\left(b_{1}, b_{2}, b_{3}\right)=(1.2570,0.9284,3.1477)$ and $\left(a_{1}, \ldots, a_{4}\right)=(-99.4002,130.8198,-58.3329,14.3040)$.

Figure 3, plots the mutual information of 16-APSK mapped symbols for different approximations. The estimated Gaussian 
mixture leads to a fine approximation of the measured mutual information at the output of the demapper.

\section{B. LDPC check and variable nodes EXIT charts}

For ease of presentation, the equalizer output LLRs will be noted as $L_{M A P, V}^{t}$ where the notation $L_{X, Y}^{t}$ designates the LLR sent form $X$ to $Y$ at iteration $t$.

\section{At iteration $t$ :}

1) A variable node $V$ of degree $i$ receives $(i+1)$ LLR messages namely $i$ check nodes LLRs $L_{C, V}^{t-1}$ following a Gaussian distribution, and the equalizer extrinsic LLR $L_{M A P, V}^{t}$ following a GM distribution. It computes then the output LLR $L_{V C}^{t}$ on the branch $n$ :

$$
L_{V C}^{t}(n)=L_{M A P, V}^{t}+\sum_{k \neq n, k=1}^{i} L_{C_{k}, V}^{t-1}
$$

The resulting LLR follow a GM distribution which is the convolution of a Gaussian and a GM distribution. More specifically,

$$
\begin{aligned}
& I_{V C}^{t}(i)= \\
& \sum_{m=1}^{M_{G}} \pi_{m} J\left(\sqrt{\beta_{m} \Psi^{-1}\left(I_{M A P, V(i)}^{t}\right)^{2}+(i-1) J^{-1}\left(I_{C, V}^{t-1}\right)^{2}}\right)
\end{aligned}
$$

where $I_{M A P, V(i)}^{t}=T\left(I_{V, M A P}^{t-1}(i)\right)$. Thus, the overall variable nodes output mutual information $I_{V, C}^{t}$ is expressed as follows:

$$
I_{V, C}^{t}=\sum_{i=2}^{d_{v}} \lambda_{i} I_{V, C}^{t}(i)
$$

It is worth noting that by taking $M_{G}=1, \beta_{m}=1$ and $\pi_{m}=1$, equation (9) simplifies to the Gaussian approximation in [6].

2) A check node $C$ of degree $j$ receives $j$ variable nodes LLRs. The output LLR at an edge $m$ is computed by a non linear combination of the input messages as follows:

$$
L_{C V}^{t}(m)=2 \tanh ^{-1}\left(\prod_{k \neq m}^{j} \tanh \left(\frac{L_{V_{k}, C}^{t-1}}{2}\right)\right)
$$

The characterisation of the pdf of the output check node LLR is not straightforward. However, by using a consistent Gaussian approximation, one can compute the mean of the output LLR and project it on the right mutual information [13]. However a simpler yet efficient approximation named 'reciprocal channel' can be used [6]. Thus, the mutual information of the check node is expressed as:

$$
I_{C, V}^{t}=1-\sum_{j=2}^{d_{c}} \rho_{j} J\left(\sqrt{j-1} J^{-1}\left(1-I_{V, C}^{t}\right)\right)
$$

TABLE II

TEST CHANNEL VOLTERRA KERNELS

\begin{tabular}{|ll|}
\hline$h_{0}^{(1)}=0.0074-i 0.0046$ & $h_{1}^{(1)}=-0.0429+i 0.0151$ \\
$h_{2}^{(1)}=0.1603-i 0.0634$ & $h_{3}^{(1)}=0.3445-i 0.1226$ \\
\hline$h_{133}^{(3)}=-0.0066-i 0.0006$ & $h_{222}^{(3)}=0.0148-i 0.0019$ \\
$h_{223}^{(3)}=-0.0066+i 0.0010$ & $h_{232}^{(3)}=-0.0177+i 0.0016$ \\
$h_{233}^{(3)}=-0.0237+i 0.0008$ & $h_{332}^{(3)}=-0.0087+i 0.0006$ \\
$h_{333}^{(3)}=-0.0108+i 0.0010$ & \\
\hline
\end{tabular}

3) A variable node $V$ of degree $i$ computes the extrinsic LLR to be forwarded to the equalizer as apriori information $L_{V, M A P}$ as follows:

$$
L_{V, M A P}^{t}(i)=\sum_{k=1}^{i} L_{C_{k} V}^{t}
$$

Thus, the associated mutual information for a variable node of degree $i$ writes as:

$$
I_{V, M A P}^{t}(i)=J\left(\sqrt{i} J^{-1}\left(I_{C, V}^{t}\right)\right)
$$

\section{OPTIMIZATION AND CODE DESIGN}

Combining equations (9), and (10), and (12), and (14), the variable to check node information at iteration $t, I_{V, C}^{t}$, writes in a parametric recursive way as:

$$
I_{V, C}^{t}=\mathcal{G}\left(\lambda(X), I_{V, C}^{t-1}, \rho(X), T()\right)
$$

This function is non linear in the mutual information $I_{V, C}$, $\rho(X)$ but linear in the variable node-degree distribution polynomial $\lambda(X)$ for fixed values of the aforementioned parameters. Thus, by fixing a distribution $\rho(X)$, which is generally considered with concentrated degrees, optimization consists of maximizing $\sum_{i} \lambda_{i} / i$ subject to the following constraints:

$$
\left\{\begin{array}{lc}
\text { Convergence } & \mathcal{G}(\tilde{\lambda}, x, \rho, T(.)) \geq x \\
\text { Proportions } & \sum_{i=2}^{d_{v}} \lambda_{i}=1 \\
\text { Stability } & \lambda_{2} \sum_{m=0}^{M_{G}} \pi_{m} e^{\beta_{m} \Psi^{-1}(T(1)) / 8} \\
& <\frac{1}{\sum_{j=2}^{d_{c}} \rho_{j}(j-1)}
\end{array}\right.
$$

For 16APSK, $T(1)=\Psi\left(\sqrt{\frac{\left.2\|h\|\right|^{2}}{\sigma_{w}^{2}}}\right)$ where $\|h\|^{2}$ is the channel energy.

\section{A. Optimization example}

In this section, we present optimization results obtained for a MAP turbo equalizer over a Volterra channel. The channel coefficients in Tab. II are excerpted from [14] and have been computed using system identification. Fig. 4 plots theachievable rate of both the 16-APSK BICM, computed using the EXIT area approximation of a soft demapper over AWGN channel, and the MAP equalizer computed also using the area integral. It also plots the optimized rates, using both the Gaussian [6] and the GM mixture approximations previously derived. The iterative receiver, achieves a gain of $1 d B$ over the non iterative MAP equalization for the non linear channel. 


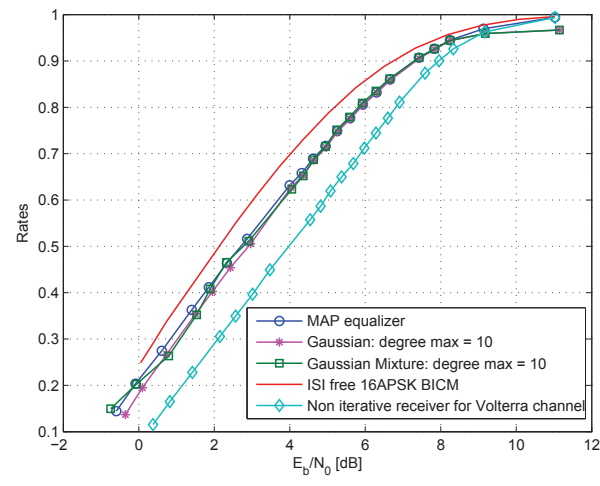

Fig. 4. Achievable designed rates compared with the MAP optimal detection and the 16-APSK ISI-free rates for a maximum $d_{v}=10$

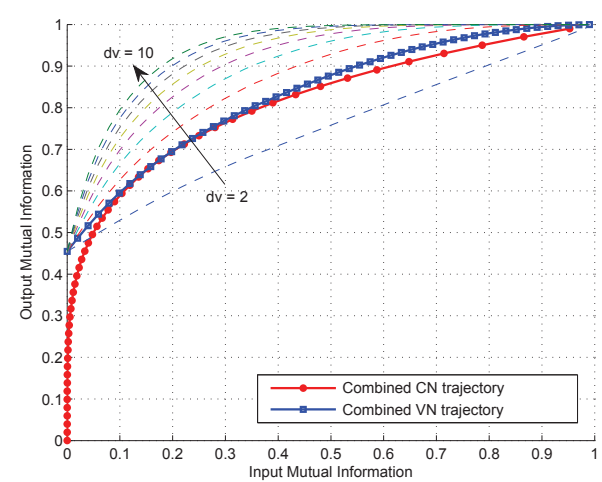

Fig. 5. Curve fitting of the Gaussian Mixture equalizer approximation

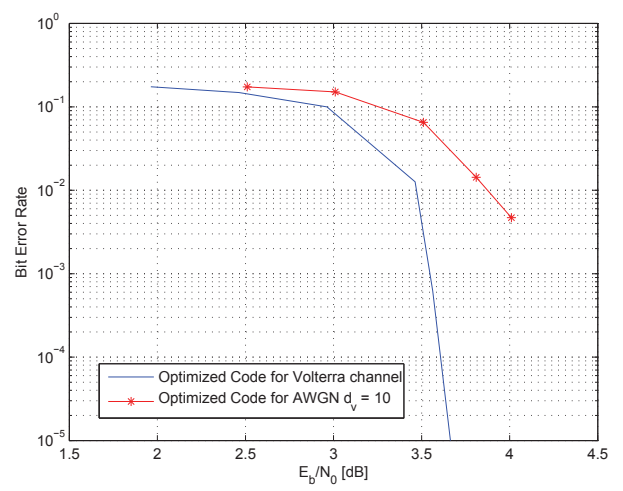

Fig. 6. Bit error rate for the iterative receiver using the optimized LDPC code

Fig. 5 plots the evolution of the average (combined) mutual information of $\mathrm{VNs}$ versus the average mutual information of CNs. The VN combined curve is computed using Equation (10) where the dashed lines represent the mutual information of different variable nodes with edge degrees $d_{v}=2$ to 10 . It can be seen from Fig. 4 that for a rate $R=0.5$, the optimized code has a threshold at $\frac{E_{b}}{N_{0}}=3.01 \mathrm{~dB}$. A code generated following the optimal degree-distribution $\lambda$ and $\rho$, should start to fall around $3 d B$. Fig. 6 plots the Bit Error Rate of a 16APSK turbo equalized with an outer LDPC code generated following the optimal degree distribution and an AWGN optimized code. Approximately $0.3 d B$ is gained optimizing the code. The estimated threshold is around $\frac{E_{b}}{N_{0}}=3.2$.

\section{CONCLUSIONS}

An iterative receiver for a non linear satellite channel was analysed and designed using a Gaussian mixture approximation of the equalizer output and partial interleaving of variable nodes. The results prove that the designed receiver achieves better performance than the non iterative optimal receiver. Besides, the study could be extended to other receiver designs namely density evolution-based, protograph codes and MET based code design.

\section{REFERENCES}

[1] C. Berrou and A. Glavieux, "Near optimum error correcting coding and decoding: turbo-codes," Communications, IEEE Transactions on, vol. 44, no. 10, pp. 1261-1271, 1996

[2] ETSI EN 302307 V1.2.1 (2009-08), "Digital video broadcasting (DVB); second generation framing structure, channel coding and modulation systems for broadcasting, interactive services, news gathering and other broadband satellite applications (DVB-S2)," European Standard (Telecommunications series), 082009.

[3] C. Douillard, M. Jezequel, and C. Berrou, "Iterative correction of intersymbol interference: Turbo equalization," European Trans. Telecommun, vol. 6, no. 5, pp. 507511, Sept.Oct 1995.

[4] A. Kavcic, Xiao Ma, and M. Mitzenmacher, "Binary intersymbol interference channels: Gallager codes, density evolution, and code performance bounds," Information Theory, IEEE Transactions on, vol. 49, no. 7, pp. 1636-1652, 2003.

[5] N. Varnica and A. Kavcic, "Optimized low-density parity-check codes for partial response channels," Communications Letters, IEEE, vol. 7, no. 4, pp. $168-170,2003$.

[6] S. ten Brink, G. Kramer, and A. Ashikhmin, "Design of low-density parity-check codes for modulation and detection," Communications, IEEE Transactions on, vol. 52, no. 4, pp. 670-678, 2004.

[7] Andrew P. Worthen and W.E. Stark, "Unified design of iterative receivers using factor graphs," Information Theory, IEEE Transactions on, vol. 47, no. 2, pp. 843-849, 2001.

[8] R. De Gaudenzi, A. Guillen i Fabregas, and A. Martinez, "Performance analysis of turbo-coded apsk modulations over nonlinear satellite channels," Wireless Communications, IEEE Transactions on, vol. 5, no. 9, pp. 2396-2407, 2006

[9] Y.T. Su, Mu-Chung Chiu, and Yen-Chih Chen, "Turbo equalization of nonlinear TDMA satellite signals," in Global Telecommunications Conference, 2002. GLOBECOM 'O2. IEEE, nov. 2002, vol. 3, pp. 2860 - 2864 vol.3.

[10] S. Benedetto, E. Biglieri, and R. Daffara, "Modeling and performance evaluation of nonlinear satellite links-a volterra series approach," IEEE Transactions on Aerospace and Electronic Systems, vol. AES-15, no. 4, pp. $494-507$, july 1979.

[11] T.J. Richardson and R.L. Urbanke, "The capacity of low-density paritycheck codes under message-passing decoding," Information Theory, IEEE Transactions on, vol. 47, no. 2, pp. 599-618, 2001.

[12] Joachim Hagenauer, "The exit chart - introduction to extrinsic information transfer," in in Iterative Processing, In Proc. 12th Europ. Signal Proc. Conf (EUSIPCO, 2004, pp. 1541-1548.

[13] K.R. Narayanan, Xiaodong Wang, and Guosen Yue, "Estimating the pdf of the sic-mmse equalizer output and its applications in designing ldpc codes with turbo equalization," Wireless Communications, IEEE Transactions on, vol. 4, no. 1, pp. 278-287, 2005.

[14] D.N. Liu and M.P. Fitz, "Iterative equalization in non-linear satellite channels," in 7th International Symposium on Turbo Codes and Iterative Information Processing (ISTC), 2012, aug. 2012, pp. 220 -224. 